\title{
Evaluation of new diagnostic procedures of medical thermography - in-vivo experiments
}

\author{
by M. Kaczmarek ${ }^{1}$, J. Rumiński ${ }^{1}$, A. Nowakowski ${ }^{1}$, A. Renkielska ${ }^{2}$, \\ J. Grudziński ${ }^{2}$, W. Stojek ${ }^{3}$
}

(1) Department of Biomedical Engineering, Technical University of Gdansk, Narutowicza 11/12, 80-952 Gdansk, Poland; phone: +48 58 3472785, fax +48 58 3471757, e-mail: mariusz@biomed.eti.pg.gda.pl,jwr@eti.pg.gda.pl, antowak@eti.pg.gda.pl;

(2) Department of Plastic Surgery and Burns, Medical University of Gdansk, Marii Sklodowskiej-Curie 3a, 80-210 Gdansk, Poland, e-mail: aren@amg.gda.pl; jgrud@amg.gda.pl,

(3) Department of Animal Physiology, Gdansk University, 80-822 Gdansk, Kladki 24, Poland,wojtek@biotech.univ.gda.pl.

\begin{abstract}
The advantages and disadvantages of active thermography in medical diagnostics using different thermal excitations: halogen lamps as a heating source and ice and a fan as cooling sources are discussed. A new procedure of tissues thermal properties identification and a new image processing method used to increase contrast between healthy and injured tissues are described. In-vivo experiments prove validity of proposed methods.
\end{abstract}

\section{Introduction}

We have shown earlier $[1,2,3]$ that the active, dynamic thermography can be applied in medical procedures for monitoring the state of the skin and subdermal tissue structure during burns treatment. It gives objective, measurable ratings of treatment procedures. Sets of burns of different degree were inducted in series of in-vivo experiments on domestic pigs in fully controlled conditions (basing on the Singer model [4]). For injury classification active thermography procedures were performed. The method proved to be non-contact, non-invasive, clean and non-stressing, allowing wide area of investigation and objective documentation for diagnostics and evaluation of a treatment process. However the value of heating excitation energy is limited by maximum acceptable skin temperature, around $42^{\circ} \mathrm{C}$, to avoid pain or any conflict with therapeutic processes. In effect the thermographic signal is rather small what is limiting accuracy of measurements.

The aim of this paper is to examine if the cooling procedure might be regarded as more suitable for burn injury diagnostics allowing the use of higher amplitudes of skin temperature changes (temperature decrease). Would it be safe, and easy in use?

For heating procedure the increment of temperature is only $6^{\circ} \mathrm{C}$ on the surface while for cooling procedures the decrement of temperature might be $30^{\circ} \mathrm{C}$ without any risk for a patient. This big difference in surface temperature change is very important for further image processing including fitting algorithms as it allows to improve signal to noise ratio. If we assume the noise at the level of $0.1^{\circ} \mathrm{C}$ (what is a typical value for most of good thermographic cameras) and temperature amplitudes for heating $\Delta T_{h}=6^{\circ} \mathrm{C}$ and for cooling $\Delta \mathrm{T}_{\mathrm{C}}=30^{\circ} \mathrm{C}$, the signal to noise ratio is improved 5 times.

\section{Organisation of experiments}

Young, weighting 20-30 kilograms, living boars were used for in-vivo experiments performed according to all rules defined in Polish law for animal experiments. Skin burn wounds of controlled depth were introduced by stamping a copper bar of the boar's back. 
In this paper we are reporting only experiments where the bar of the temperature $90^{\circ} \mathrm{C}$ was in touch with the skin for 5,10 and 15 seconds. Animals were in full anaesthesia during the burn injury. For reference data the full-thickness biopsies followed by histopathologic analysis were performed, to know exactly the degree of inflicted burns. The thermographic measurements were taken 5 hours after the burn was inflicted and then every 24 hours during the consecutive 3 days.

The general arrangement of the measurement system is typical for active thermography experiments $[1,3]$. We use the AGEMA Thermovision 900 thermographic system. The thermal resolution of the system is $0.1^{\circ} \mathrm{C}$.

For heating we use a set of halogen lamps as an excitation source. The power and time of irradiation is controlled in a big span of values. In this experiment the pulsed mode of irradiation and temperature measurements in the cooling phase were performed. Momentary maximal temperatures at the irradiated, tested surfaces were not exceeding $48^{\circ} \mathrm{C}$.

For cooling the ice was applied, hold in a thin plastic bag in touch with a tested tissue for 10 seconds. Thermographic measurements were taken during the recovery time while self-heating of the tissue is dominant, after taking the ice off.

For cooling we applied also an air fan allowing undisturbed thermal registration during the cooling as well as at the heating phases. It seems to be a very convenient and safe technique giving very promising results. The phase of forced cooling is followed by recovery to the steady conditions, what in this paper is called "natural heating".

The start of registration and the parameters of thermographic sequences (repetition rate and the end of registration time) are dependent on an excitation method and tissue temperature recovery mechanism. Depending on a case the speed of registration varies from several frames per second to one frame every several seconds.

\section{Identification procedure}

The way of application of a thermal excitation as well as thermal properties of tested tissue play the major role for a shape of the observed temperature profiles - see Fig. 1 . Assuming for living tissue that only the recovery phase following an excitation is observed we may study the cooling process after optical heating excitation or the rise of temperature after cooling in the case of the ice stress. For the air fan method both cooling and rising phases - can be observed. Two measurable processes - heating and cooling - may be related to different physiological phenomena. The observed dynamic processes leading to equilibrium after pulse excitation (heating or cooling) may be characterised by different time constants. For the two phases the equivalent thermal models are described by different equations. Assuming the two-exponential model one may get the time dependence of skin surface temperature as:

$$
T(t)=T_{\min }+\Delta T_{1} e^{-\frac{t}{\tau_{1 c}}}+\Delta T_{2} e^{-\frac{t}{\tau_{2 c}}}
$$

for the natural cooling process (after external heating to $T_{\max }=T_{\min }+\Delta T_{1}+\Delta T_{2}$, where

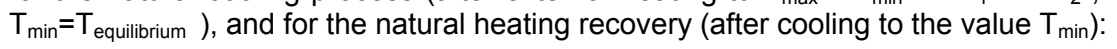

$$
T(t)=T_{\min }+\Delta T_{1}\left(1-e^{-\frac{t}{\tau_{1} h}}\right)+\Delta T_{2}\left(1-e^{-\frac{t}{\tau_{2} h}}\right)
$$

where: $\tau_{1}, \tau_{2}$ - time constants, $c$ - indicates the cooling and $h$ - indicates the heating phase.

The two components thermal model are applied here to calculate the complex surface temperature profiles of a two-layer medium. Due to limited accuracy of measured temperature changes (small signal to noise ratio) for some cases it might be sufficient to 
use only the one layer model - expressed by one exponent factor. In recorded pictures, the differences between pixels of different thermal properties are displayed during the observation time as in the example shown in Fig. 2b. For the acquired data the fitting curve procedure using the Levenberg-Marquardt or the Gauss-Newton algorithm is applied to estimate the equivalent model parameters. Some examples of fitting the data to the one component exponential model for the described excitations are presented in Table 1. One can differentiate between healthy and burned tissues basing on values of time constants $\tau$. For burned tissue the values are correlated with the burn depth.

For comparing the efficiency of proposed excitation methods and for classification of investigated tissues an objective measure is needed. We define here, as the basic figure of merit for each pixel $i$, the injury index $-\| I^{\prime}$ :

$$
I^{i}=\frac{\tau^{i}-\tau^{r e f}}{\tau^{i}+\tau^{r e f}}
$$

where: $i$ - time constant at the pixel $i$, and $\tau^{\text {ref }}$ the time constant at the healthy area taken as a reference. The reference area should be placed as close to the burned tissue as it is possible or might be taken from a symmetrical point of a tested body. The injury index is close to zero for tissues of thermal properties similar to the healthy tissue. It falls down to minus 1 for tissues characterised by rapid heat exchange, e.g. the places of high vascularisation caused by light superficial burns. It rises up to 1 for tissues characterised by slow heat exchange, what is the case of deep burns.

Injury index allows discrimination of the state of the injured skin (depth of burns).

To compare efficiency of different excitation methods the synthetic image of $\tau_{1}$ should be calculated. After application of segmentation procedure the new image is composed of $n$ regions of different thermal properties, which are related to the estimated values of the time constants. As an additional figure of merit the dispersion coefficient $D$ may be defined basing on the formula:

$$
D=\sqrt{\frac{n \sum_{j=1}^{n}{\overline{\tau^{j}}}^{2}-\left(\sum_{j=1}^{n} \overline{\tau^{j}}\right)^{2}}{n(n-1)}}
$$

where: $n$ - the number of regions discriminated in the synthetic image of $\tau_{1}$ after segmentation; $\overline{\tau^{j}}$ - the mean value of a time constant in the region $j$. The formula is using the same concept as the standard deviation and is showing dispersion of the values of time constants. The resolution of the segmented image is dependent on the accuracy of measurements performed, leading to discrimination of a higher number $n$. The dispersion coefficient $D$ calculated for all methods of excitation is presented in Table 1 and Fig. 3 . The best results are obtained for the ice stress method (the biggest dispersion coefficient), comparable results are for the both phases (cooling and heating) of the air fan method. The worst is the optical (halogen) heating method due to limited temperature rise after excitation. In this case the time constant values for different degrees of injury are close to each other, the dispersion coefficient is of the smallest value. The presented data for different excitations were taken in different places of injury therefore the conclusions given here should be proved in additional series of experiments.

\section{Results}

The data in Table 1 are obtained for arbitrary chosen characteristic Control Points of burn wounds shown in Fig. 2a. One layer equivalent thermal model (one exponent factor) equation (1) is applied. Instead of temperature the emitted radiation R in arbitrary units are taken for calculations. The Control Point 1 is characterised by burn conditions: the bar 
temperature $90^{\circ} \mathrm{C}$ for 15 seconds contact with skin; the Control Point 2 - the bar temperature $90^{\circ} \mathrm{C}$ for 10 seconds contact with skin, etc. The Control Point 4 is the reference point representing a healthy skin.

Table 1: Estimated model parameters for different tissue excitations

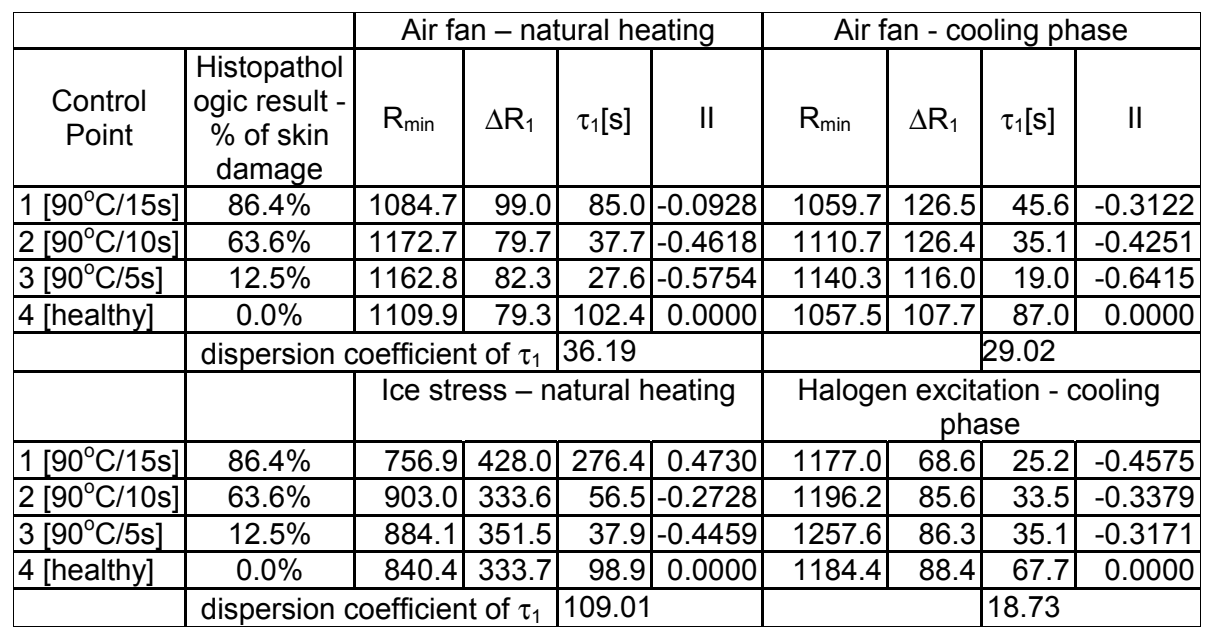

Taking into account the set of model parameters the time constant $\tau$ seems to be the best for tissue classification. For the ice stress cooling procedure the time constant $\tau_{1}$ of a healthy tissue is almost two times smaller comparing to the time constant characteristic for the full thickness burn.This can be explained as an effect of destruction of the microcirculation blood system for the III-rd degree burns. The heat transport to the skin surface is in this case poor. Therefore to return to the equilibrium temperature, it takes longer time then for the healthy tissue or for a tissue under a healing process, characterised by increased blood circulation. Figs. 4a-d) show estimated time constants $\tau_{1}$ for the whole area of investigation and for all excitation methods.

The injury index synthetic pictures for the air fan method are shown in Fig. $5 a$ and b. The dark areas (the injury under a healing process) on both pictures are slightly different. In the first picture sharp edges of injuries are clearly seen while in the second one the injury areas are bigger and diffused. It can be explained by the difference of heat transport mechanism. The forced air-cooling is a quite fast process and the heat flow is mainly twodimensional. Heat exchange is possible only by a thin film of tissues on the surface while for natural heating process the heat flow is slower and the 3-dimensional heat exchange, with big influence of deeper tissues should be taken into account. One can see in Fig. $2 b$ that after recovery time lasting 4 minutes (the same as for the cooling phase) surface tissues are still cooler then at the equilibrium.

\section{Conclusions}

Discrimination between time constants characteristic for the heating and cooling phases needs some more investigation and study of basic physiological processes. Thermal excitation influences conditions of heat exchange. The better thermal contact and the lower thermal resistance the faster thermal response! Ice will be cooling faster than air stream; radiation will be dependent on temperature differences between a tested surface and the environment, etc. Therefore here we only claim that it is possible to quantify and classify a burn injury and indicate affected tissue surface basing on visualisation of the 
time-constants and injury index distribution. We see a special possibility of using the air cooling with controlled temperature in wide range - from lower to high temperatures. It allows to use higher amplitudes of external excitation similar to those used in the ice stress method but the air method is fully non-contact and septic. The cooling methods seem to be better from the therapeutic point of view in burns diagnostics than the heating as the external heating of tissues may additionally destroy deeper layers of the skin.

The measurement starting moment, promptly after temperature excitation, is of the highest significance for proper registration of thermograms. The other problem is the time period between injury and diagnostic examination. To our experience the most valuable results are obtained during the first and the second days following an accident. The clinical most valuable feature of the method is determination of the depth of a burned tissue. Still more experiments to set the thresholds of injury index for classification of tissues for surgical or pharmacological treatment are necessary.

\section{Acknowledgement}

A part of this work was financed by the KBN research grant 7T11E 01521. The authors thank for the technical help to Mr A. Galikowski.

\section{REFERENCES}

[1] NOWAKOWSKI A., KACZMAREK M., RUMINSKI J., HRYCIUK M., RENKIELSKA A., GRUDZINSKI J., SIEBERT J., JAGIELAK D., ROGOWSKI J., ROSZAK K., STOJEK W., Medical applications of model based dynamic thermography, Thermosense XXIII Proc. of SPIE, vol. 4360, 2001, p. 492-503

[2] RUMIŃSKI J., KACZMAREK M., NOWAKOWSKI A., Medical Active Thermography - A New Image Reconstruction Method, Lecture Notes in Computer Science, LNCS 2124, Springer, Berlin-Heidelberg, 2001, p. 174-181

[3] KACZMAREK M., NOWAKOWSKI A., RENKIELSKA A., Rating burn wounds by dynamic thermography, Proc. of QIRT 2000, 2000, p.376-381

[4] SINGER A.J., BERRUTI L., THODE HC J.R., MCCLAIN S.A., Standardised burn model using a multiparametric histologic analysis of burn depth, Academic Emergency Medicine, vol. 7:1, 2000, p. 1-6 
a)

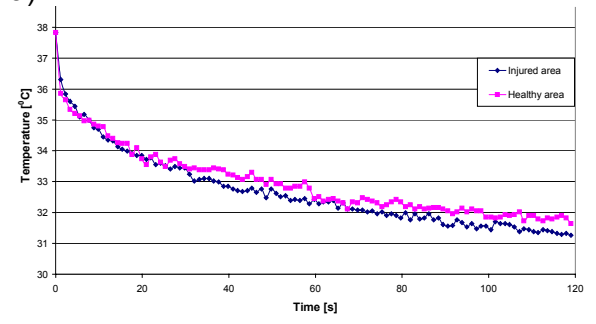

b)

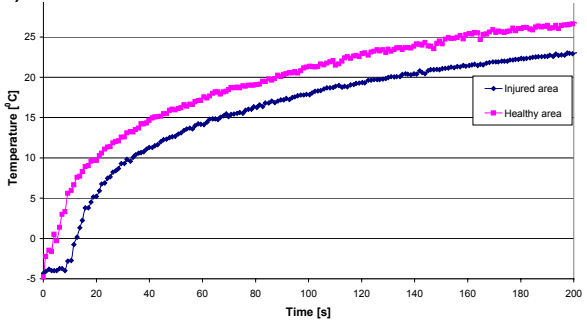

Fig. 1. Time response of injured and healthy tissues; a) for the optical heating method; b) for the ice stress method

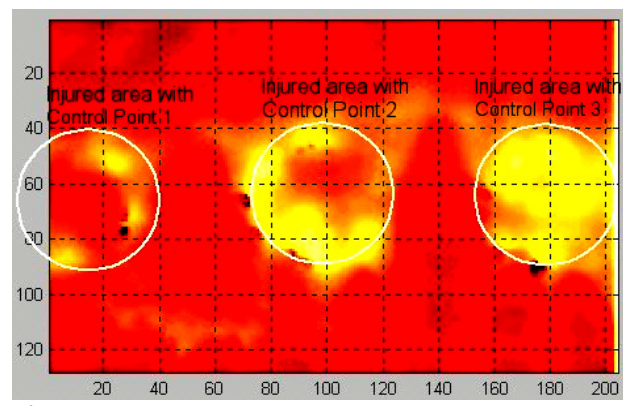

a)

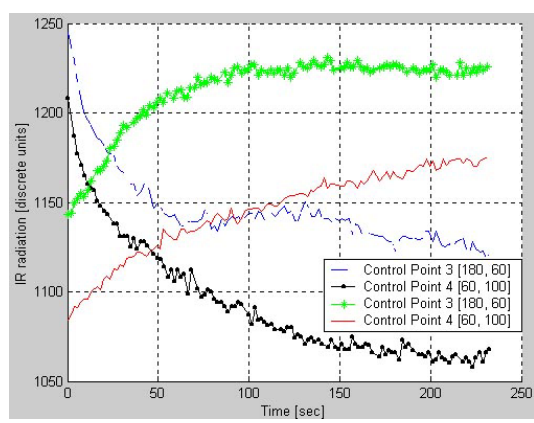

b)

Fig. 2. a) Investigation areas - details in Table 1, b) Time response of injured and healthy tissues in Control Points 3 and 4 for the air fan method (cooling and heating phase)

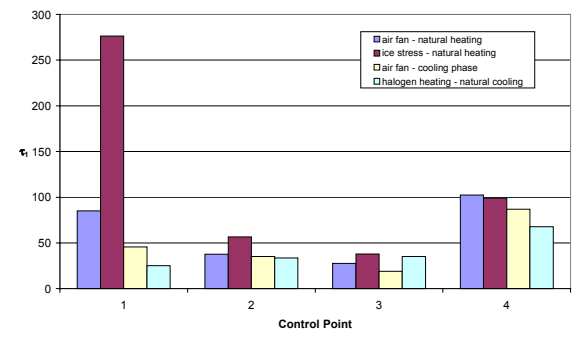

a)

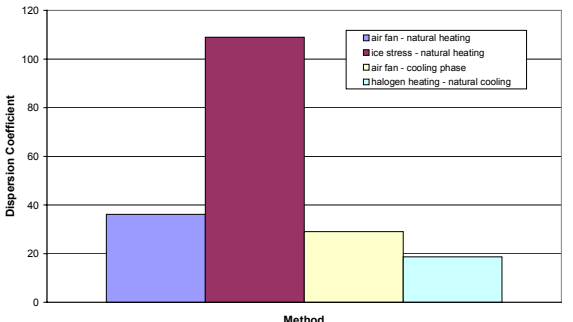

b)

Fig. 3. a) Estimated time constant $\tau_{1}$ for different excitations, b) the dispersion coefficient calculated for different excitations 
a)
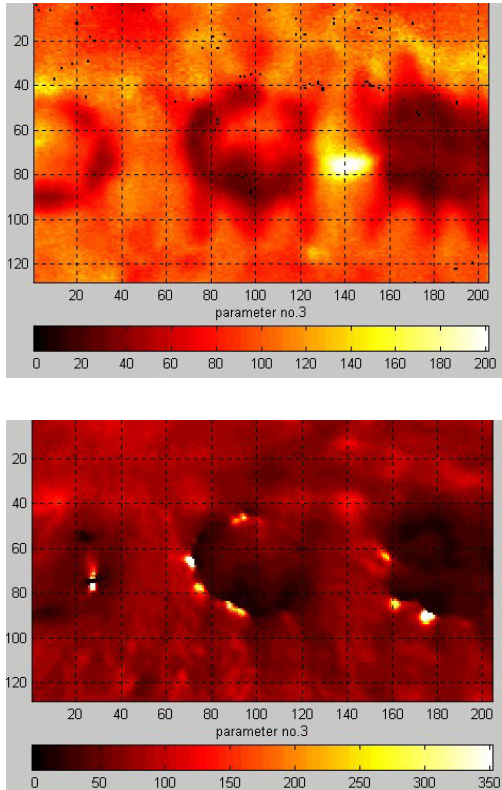

c)

Fig. 4. Synthetic images of estimated $\tau_{1}$ parameter for: a) the air fan natural heating phase, b) the ice stress - natural heating phase, c) the air fan - cooling phase, d) the halogen heating - cooling phase; all done for second day investigation

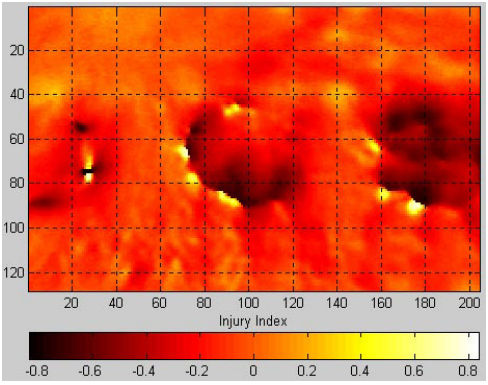

a)
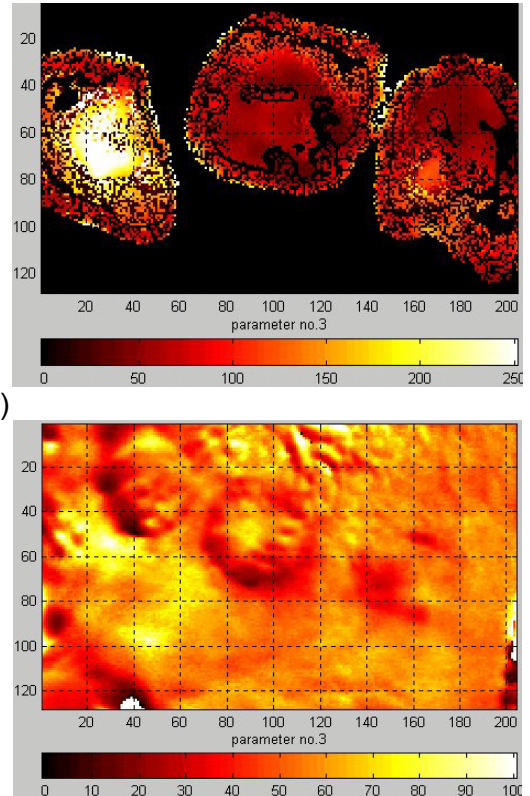

d)
Fig. 5. Injury index images for the air fan method for investigation done

48 hour following the injury creation; a) cooling phase; b) natural heating phase b)

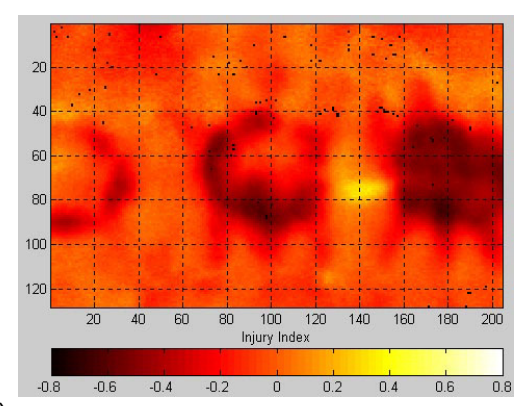

